\title{
Different correlations among physiological and morphological properties at single glutamatergic synapses in the rat hippocampus and the cerebellum.
}

\section{$\operatorname{AUTHOR(S):~}$}

Miyawaki, Hiroyuki; Hirano, Tomoo

\section{CITATION:}

Miyawaki, Hiroyuki ...[et al]. Different correlations among physiological and morphological properties at single glutamatergic synapses in the rat hippocampus and the cerebellum.. Synapse 2010, 65(5): 412-423

\section{ISSUE DATE:}

2010-10-20

URL:

http://hdl.handle.net/2433/197147

\section{RIGHT:}

This is the peer reviewed version of the following article: Miyawaki, H. and Hirano, T. (2011), Different correlations among physio logical and morphological properties at single glutamatergic synapses in the rat hippocampus and the cerebellum. Synapse, 65: 412-423, which has been published in final form at http://dx.doi.org/10.1002/syn.20860; この 論文は出版社版でありません。引用の際には出版社版をご確認ご利用ください。; This is not the published version. Please cite only the published version. 
Different Correlations among Physiological and Morphological Properties at Single Glutamatergic Synapses in the Rat Hippocampus and the Cerebellum

Hiroyuki Miyawaki and Tomoo Hirano

Department of Biophysics, Graduate School of Science, Kyoto University,

Kitashirakawa-Oiwake-cho, Sakyo-ku, Kyoto 606-8502, Japan

Running title: Correlations of Single-Synapse Properties

Correspondence should be sent to Tomoo Hirano,

Department of Biophysics, Graduate School of Science, Kyoto University,

Kitashirakawa-Oiwake-cho, Sakyo-ku, Kyoto 606-8502, Japan.

Tel: 81-75-753-4237, Fax: 81-75-753-4229

Email: thirano@neurosci.biophys.kyoto-u.ac.jp

Keywords

quantal EPSC, presynaptic varicosity, spine, culture 


\section{Abstract}

Synapses in the mammalian central nervous system show substantial diversity in their physiological and morphological properties. However, the correlations among them have remained elusive. Here, we tried to clarify the correlations by establishing a method to record excitatory postsynaptic currents (EPSCs) at individual synapses and also to observe the morphology at the same time. A pair of pre- and postsynaptic neurons were labeled with different fluorescent dyes, and a presynaptic varicosity was selectively stimulated with a $\theta$-tube glass electrode under conditions in which action potential generation was suppressed. Two representative types of excitatory glutamatergic synapses, one on hippocampal pyramidal neurons and the other on cerebellar Purkinje neurons, were studied. The correlations between the properties of quantal EPSCs (qEPSCs) and those of synaptic morphology were analyzed in rat primary culture preparations. The amplitude and the decay time of qEPSC were correlated with the size of the postsynaptic spine only at hippocampal synapses. In contrast, the size of the presynaptic varicosity was correlated with the size of the postsynaptic spine and the quantal content of evoked EPSCs only at granule neuron - Purkinje neuron synapses in the cerebellum. These results suggest that the interaction between pre- and postsynaptic structures and the coupling of postsynaptic responsiveness and the spine 
morphology differ between cerebellar and hippocampal glutamatergic synapses. 


\section{Introduction}

Glutamate is the predominant excitatory transmitter in the mammalian central nervous system. Among glutamatergic synapses, those on hippocampal pyramidal neurons and those on cerebellar Purkinje neurons have been studied extensively (Bekkers et al., 1990; Christie and Jahr, 2006; Conti and Lisman, 2003; Denk et al., 1995; Dobrunz and Stevens, 1997; Foster et al., 2005; Futai et al., 2007; Harris and Stevens, 1988, 1989; Hirano and Hagiwara, 1988; Liu and Tsien, 1995; Matsuzaki et al., 2004; Okamoto et al., 2004; Sdrulla and Linden, 2007; Tanaka et al., 2007), and distinct properties of these two types of synapses have been reported. First, synapses on hippocampal pyramidal neurons express not only a-amino-3-hydroxyl-5-methyl-4-isoxazolepropionic acid receptors (AMPARs) but also $\mathrm{N}$-methyl-D-aspartic acid receptors (NMDARs) as postsynaptic ionotropic glutamate receptors. Activation of NMDARs causes a large $\mathrm{Ca}^{++}$influx and triggers long-term potentiation (LTP) of synaptic responses. LTP is accompanied by an increase in the postsynaptic spine size, and long-term depression (LTD) is accompanied by a decrease (Matsuzaki et al., 2004; Okamoto et al., 2004; Zhou et al., 2004). In contrast, NMDARs are not expressed at cerebellar granule neuron - Purkinje neuron synapses (Hirano and Hagiwara, 1988), and LTD occurs without a concomitant shrinkage of spine sizes at 
these synapses (Sdrulla and Linden, 2007). Physiological and morphological heterogeneity has also been reported within a single type of synapse. Sizes and shapes of postsynaptic spines, and the amplitudes and the time courses of electrophysiological postsynaptic responses vary substantially in a single type of synapse (Harris and Landis, 1986; Lee et al., 2004; Murphy et al., 1995; Nusser et al., 1997, 1998). Some correlations among the properties of synapses have been reported. For example, synapses further from a soma tend to express larger numbers of AMPARs in hippocampal pyramidal neurons, cancelling the electrotonic attenuation of EPSP amplitudes recorded at the soma (Andrasfalvy and Magee, 2001; Magee and Cook, 2000; Nicholson et al., 2006; Smith et al., 2003). However, the detailed correlations between the structure and function of central synapses remain elusive.

In this study, we tried to measure various physiological and morphological properties of individual excitatory synapses and clarify the correlations among them, focusing on the two representative types of glutamatergic synapses, one on hippocampal neurons and the other on cerebellar Purkinje neurons. To this end, we established a method to record EPSCs at an individual synapse and to observe the morphology of both a presynaptic varicosity and a postsynaptic spine at the same time. We labeled pre- and postsynaptic neurons with different fluorescent dyes, and applied electrical stimulation selectively to 
a visually identified presynaptic varicosity under conditions in which action potential generation was suppressed. We also developed an objective method to estimate qEPSC based on the similarity of EPSC wave forms. These methods unveiled distinct correlations among morphological and physiological properties at the two types of central glutamatergic synapses.

\section{Materials and Methods}

Culture

Methods used to prepare primary cultures of hippocampal neurons were similar to those previously described (Brewer et al., 1993). In brief, pregnant Wistar rats were killed by cervical dislocation, and day 18 or 19 embryos were taken out and decapitated. Then, hippocampi were dissected out from the embryos, and incubated in $\mathrm{Ca}^{++}$and $\mathrm{Mg}^{++}$ free Hanks' balanced salt solution containing 0.1\% trypsin and 0.05\% DNase for $15 \mathrm{~min}$ at $37^{\circ} \mathrm{C}$. Cells were dissociated by trituration and seeded on poly-D-lysine-coated coverslips in Neurobasal Medium (Invitrogen, Carlsbad, CA, United States) containing $0.5 \mathrm{mM}$ L-glutamine, $25 \mu \mathrm{M}$ L-glutamate, and $2 \% \mathrm{~B} 27$ supplement (Invitrogen). Three days after dissociation, half of the medium was replaced with glutamate-free medium. Thereafter, half of the medium was replaced by the fresh medium every 7 days. 
Electrophysiological experiments were performed 13 - 19 days after dissociation.

Methods of preparing primary cultures of cerebellar neurons were similar to those previously described (Hirano and Kasono, 1993; Kawaguchi and Hirano, 2006). In brief, pregnant Wistar rats were killed by cervical dislocation. Embryos or newborn rats were decapitated, and the cerebella were taken out and incubated in $\mathrm{Ca}^{++}$and $\mathrm{Mg}^{++}$free Hanks' balanced salt solution containing $0.1 \%$ trypsin and $0.05 \%$ DNase for 15 min at $37^{\circ} \mathrm{C}$. Cells were dissociated by trituration and seeded on poly-D-lysine-coated coverslips in Dulbecco's modified Eagle medium/F12-based medium containing 2.5\% fetal bovine serum (Furuya et al., 1998). About 24 hours after dissociation, $75 \%$ of the medium was replaced with serum-free Eagle-based basal medium. Thereafter, half of the medium was replaced every 5 days. To inhibit glial proliferation, $4 \mu \mathrm{M}$ cytosine B-D-arabino-furanoside (Sigma, St. Louis, MO, United States) was added to the medium from 1 week after dissociation. Electrophysiological experiments were performed on cerebellar neurons kept in culture for $18-24$ days.

Experimental procedures were in accordance with the guidelines regarding care and use of animals for experimental procedures of the National Institutes of Health (United States) and Kyoto University, and approved by the local committee for handling experimental animals in the Graduate School of Science, Kyoto University. 
DNA transfection

The pCAsalEGFP (Horikawa and Takeichi, 2001; Mizuno et al., 2007) and the pCAG-TurboRFP (Mizuno et al., 2010) plasmids were obtained from H. Mizuno, and a plasmid encoding EGFP and adeno-associated viral vector (AAV) from M. Kengaku. Lipofection with LipofectAMINE2000 (Invitrogen) was performed to transfect the expression vector into cultured hippocampal neurons $3-7$ days after dissociation. Electroporation (using a Nucleofector device and a Rat Neuron Nucleofector Kit, Lonza Cologne AG, Cologne, Germany) was used to transfect the expression vector into cerebellar granule neurons before seeding. To express EGFP in cerebellar Purkinje neurons, AAV was used. The plasmid encoding EGFP and AAV was transfected into HEK293 cells using $\mathrm{Ca}^{++}$phosphate. The AAV was purified from the transfected HEK293 cells with an AAV Purification ViraKit (Virapur, LLC, San Diego, CA, United States), and it was added to the cerebellar culture 1 day after dissociation.

Live imaging of synapses

Neurons with synaptic contacts were visualized with EGFP, and Alexa594 or turboRFP.

All images were acquired with a laser scanning confocal microscope (FV500, Olympus, 
Tokyo, Japan) equipped on an inverted microscope (IX 71, Olympus) with x60 objective lens (PLAPON 60XO, NA 1.42, Olympus). EGFP was excited with a $488 \mathrm{~nm}$ laser and the emitted light with wavelength between $505 \mathrm{~nm}$ and $525 \mathrm{~nm}$ was regarded as the fluorescent signal. Alexa594 or TurboRFP was excited with a $543 \mathrm{~nm}$ laser and the emitted light with wavelength $>610 \mathrm{~nm}$ or $>560 \mathrm{~nm}$ was regarded as the signal, respectively. Several images at different focal planes were captured with an interval of $0.25 \mu \mathrm{m}, 0.5 \mu \mathrm{m}$ or $1 \mu \mathrm{m}$.

The distance between the stimulated synapse and the soma was measured by drawing a line along the dendrite. The presynaptic terminal region was outlined on a $\mathrm{z}^{\text {-stacked }}$ image and the size was measured. The border of the presynaptic area was determined as follows. The sum of the mean plus twice the S.D. of background signals was regarded as noise, and was subtracted from the original image. Then, $20 \%$ of the maximum signal intensity value of the varicosity was set as the threshold.

The fluorescent signal intensity of dendritic shafts was much stronger than that of the spines. Thus, a simple thresholding method was unable to separate the dendritic shaft and a postsynaptic spine clearly, and determination of the spine boundary was difficult in some cases. This problem was more critical in cerebellar Purkinje neurons with thicker dendrites and higher spine densities. Therefore, to outline the postsynaptic 
spine region, we adopted the following method. The confocal image was low-pass filtered, and the sum of the mean plus two S.D.s of background signals was regarded as noise. This noise was subtracted from the image, and $50 \%$ of the maximum signal intensity value of the spine was set as the threshold. Then, the pixel with the maximum signal intensity in a spine was selected. If the signal intensity of a pixel next to the selected pixel was larger than the threshold and not larger than the signal intensity of the selected pixel, it was included in the selected region. This procedure was performed in all $\mathrm{x}^{-}, \mathrm{y}^{-}$and $\mathrm{z}^{-}$axis directions. Thus, 6 adjacent pixels were checked in each step. This procedure was repeated as long as any pixels were added to the selected region. At the end, the spine area was confirmed visually, and its size was measured after stacking the selected region into a plane. These morphological analyses were performed with ImageJ (National Institutes of Health; http://rsb.info.nih.gov/ij/). Three-dimensional images of synapses were reconstructed with ImageSurfer (Center for Computer-Integrated Systems for Microscopy and Manipulation, and Department of Cell and Developmental Biology, The University of North Carolina; http://imagesurfer.cs.unc.edu/).

\section{Electrophysiology}

Whole-cell patch clamp recording was performed on hippocampal pyramidal neurons or 
on cerebellar Purkinje neurons with an amplifier (EPC10, HEKA Elektronik, Lambrecht/Pfalz, Germany) in a solution containing (in mM) $145 \mathrm{NaCl}, 5 \mathrm{KOH}, 2 \mathrm{CaCl}_{2}$, $1 \mathrm{MgCl}_{2}, 10$ glucose and 10 Hepes (adjusted to $\mathrm{pH} 7.3$ with $\mathrm{NaOH}$ ) at room temperature $\left(21-25{ }^{\circ} \mathrm{C}\right)$. The solution also contained $1 \mu \mathrm{M}$ tetrodotoxin (TTX; Wako, Osaka, Japan) to suppress action potentials and $20 \mu \mathrm{M}$ bicuculline (Tocris Bioscience, Bristol, UK) to suppress GABAergic inhibitory postsynaptic currents. A glass patch pipette (2- $7 \mathrm{M} \Omega)$ was filled with intracellular solution containing (in mM) $150 \mathrm{CsCl}, 15 \mathrm{CsOH}, 5$ EGTA, 2 Mg-ATP (Sigma), 0.2 Na-GTP (Sigma) and 10 Hepes (adjusted to pH 7.3 with $\mathrm{CsOH}$ ). To label a postsynaptic neuron, $80 \mu \mathrm{M}$ Alexa Fluor 594 hydrazide (Invitrogen) was also added. The membrane potential was held at $-70 \mathrm{mV}$. The signal was filtered at $2.9 \mathrm{kHz}$ and digitized at $20 \mathrm{kHz}$. Only recordings with the input resistance of $>100 \mathrm{M} \Omega$ and leak current of $<500 \mathrm{pA}$ were accepted, and an experiment was terminated when the series resistance changed by $>25 \%$. A stimulating glass $\theta$-tube electrode (Chen et al., 2004; Fedulova et al., 1999; Kirischuk et al., 1999) was placed very close to a visually identified presynaptic varicosity, and $500 \mu \mathrm{s}$ voltage pulse was applied. The threshold stimulation voltage to evoke a synaptic response was determined by changing voltage between 0 and $30 \mathrm{~V}$. In most cases, the intensity of stimulation was $10-20 \mathrm{~V}$. The stimulation was applied at $0.2-0.5 \mathrm{~Hz}$. 


\section{FM1-43 measurement}

Presynaptic varicosities were labeled with FM1-43 (Invitrogen) in $50 \quad \mu M$ FM1-43-containing high $\mathrm{K}^{+}$solution $(95 \mathrm{mM} \mathrm{NaCl}, 50 \mathrm{mM} \mathrm{KCl}, 5 \mathrm{mM} \mathrm{NaOH}, 2 \mathrm{mM}$ $\mathrm{CaCl}_{2}, 1 \mathrm{mM} \mathrm{MgCl} 2,10 \mathrm{mM}$ glucose and $10 \mathrm{mM}$ Hepes, pH 7.3 with $\left.\mathrm{NaOH}\right)$ ) for 1 minute (Murthy et al., 1997; Stevens and Williams, 2000). The extracellular FM1-43 was then washed-out with normal extracellular solution containing $1 \mathrm{mM}$ ADVASEP-7 (Biotium Inc, Hayward, CA, United States), $1 \mu \mathrm{M}$ TTX, and $20 \mu \mathrm{M}$ bicuculline for $>3$ minutes. The $\theta$-tube glass stimulating electrode was placed adjacent to a varicosity, and an electrical pulse $(500 \mu \mathrm{s}, 30 \mathrm{~V})$ was applied 100 times at $10 \mathrm{~Hz}$ to unload FM1-43. Then 90 seconds after the stimulation, the high $\mathrm{K}^{+}$solution was applied to confirm that remaining FM1-43 signals would be unloaded. FM1-43 was excited by a $488 \mathrm{~nm}$ laser and the emitted light with wavelength $>560 \mathrm{~nm}$ was regarded as the signal. FM1-43 images were captured at about 22.4 frames per minute. The intensity of the FM1-43 signal was analyzed in 3 areas: $<2 \mu \mathrm{m}, 2-5 \mu \mathrm{m}$, and $5-10 \mu \mathrm{m}$ distant from the center of the stimulating electrode tip. The analysis was performed with ImageJ (National Institutes of Health). 


\section{Estimation of quantal EPSC}

Dissimilarity $\left(\mathrm{D}_{\mathrm{i}, \mathrm{j}}\right)$ between two EPSCs $\left(\mathrm{EPSC}_{\mathrm{i}} \text { and } \mathrm{EPSC}_{\mathrm{j}}\right)_{\text {was defined as }}$

$$
\mathrm{D}_{i, j}=\sqrt{\sum_{t}\left(E_{i}(t)-E_{j}(t)\right)^{2}}
$$

Here, $E_{i}(t)$ and $E_{j}(t)$ are the current amplitudes of $\mathrm{EPSC}_{i}$ and $\mathrm{EPSC}_{\mathrm{j}}$ at time $\mathrm{t}$. To calculate dissimilarity, we used current data acquired between $0.75 \mathrm{~ms}$ and $25 \mathrm{~ms}$ after the stimulus (485 points). Dissimilarities were calculated among all EPSCs recorded in the same stimulation condition. Based on the dissimilarities, a hierarchical cluster analysis of EPSCs was performed with Ward's minimum variance method (Ward, 1963). EPSCs were classified into several groups by cutting the dendrogram at a threshold value of $200 \mathrm{pA}$, which corresponded approximately to the sum of the mean plus two S.D.s of dissimilarities among failure events. The EPSC groups containing $<3$ events were discarded. Then each averaged EPSC was low-pass $(1 \mathrm{kHz})$ filtered and the peak time was measured. If there was a $>0.5 \mathrm{~ms}$ difference in the peak times among different EPSC groups, data were discarded. We presumed that remaining EPSCs in these groups were composed of a single type of qEPSCs. Either the smallest averaged EPSC of a group or the smallest difference trace between the averaged EPSCs was tentatively regarded as a qEPSC. The integral number obtained by dividing the amplitude of the averaged EPSC by the tentative qEPSC amplitude was regarded as the quantal content 
of each group. If one of the divided values differed from an integral number by $>0.2$, the data were discarded. Finally, the qEPSC wave-form was obtained by averaging EPSC traces divided by their respective quantal contents. These analyses were performed with R (R Foundation for Statistical Computing; http://www.r-project.org/).

\section{Statistics}

All data were expressed as mean \pm S.D. unless otherwise stated. All statistical analyses were performed with R (R Foundation for Statistical Computing).

\section{Results}

\section{Recording EPSCs at a morphologically identified synapse}

To examine the morphological and physiological properties of single synapses and to clarify their correlations, we developed a method to locally stimulate a visualized presynaptic varicosity apposed to a postsynaptic spine in neuronal culture preparations. Presynaptic neurons were labeled with EGFP, and a postsynaptic neuron was stained with Alexa594 introduced from a glass electrode used for whole-cell patch-clamp recording. These procedures visualized both presynaptic axons and postsynaptic dendrites and spines. Usually, there are many synaptic contacts between an axon and a 
postsynaptic neuron. Therefore, activation of an axon should induce transmitter release from multiple presynaptic varicosities. To restrict the number of activated presynaptic varicosities, action potential generation was prevented by adding TTX to the extracellular solution, and local electrical stimulation was applied through a $\theta$-tube glass stimulation electrode placed very close to a presumptive presynaptic varicosity.

We first applied this method to glutamatergic synapses on pyramidal neurons in a dissociated hippocampal culture preparation. Fig. 1A and 1B show axons of presynaptic neurons (green) and a pyramidal neuron (magenta). Higher magnification views showed that there was only one presumptive synaptic contact between a varicosity and a spine in this area (Fig. 1B,C). Electrical stimulation of the presumptive presynaptic varicosity evoked small EPSCs (Fig. 1D). The intensity of electrical stimulation was set at a threshold level, so that failure events were observed. The recorded EPSCs showed a similar time course (Fig. 1D), and there were two peaks (6 pA, and $11 \mathrm{pA})$ in the amplitude histogram (Fig. 1E). The larger peak amplitude was approximately twice the amplitude of the smaller peak, suggesting that the recorded EPSCs were composed of qEPSCs whose amplitude was approximately $5-6 \mathrm{pA}$. These results suggest that only a single presynaptic varicosity was activated in this case.

To study individual synaptic properties, it is critical to confirm that the effect of 
stimulation is confined to one synapse. To estimate the area where the electrical stimulation was effective, we examined the release of FM1-43 dye from presynaptic varicosities. FM1-43 was loaded into presynaptic varicosities by treatment with a high $\mathrm{K}^{+}$solution supplemented with FM1-43, and then electrical stimulation pulses were applied. We used $30 \mathrm{~V}$ pulses, which corresponded to the strongest intensity used to induce EPSCs in this study. The electrical stimulation destained the FM1-43 signals located within $2 \mu \mathrm{m}$ from the center of the glass electrode tip, but did not destain the signals located $>2 \mu \mathrm{m}$ away (Fig. 2). Most of FM1-43 signals were destained by the following treatment with a high $\mathrm{K}^{+}$solution which would depolarize all neurons, confirming that the FM1-43 signals came from presynaptic varicosities. These results suggest that the electrical stimulation with a $\theta$-tube glass electrode caused the release of synaptic vesicles only from presynaptic varicosities located within $2 \mu \mathrm{m}$ from the center of the stimulation electrode tip. In most EPSC recordings, the intensity of stimulation was $10-20 \mathrm{~V}$, suggesting the spread of effective electrical stimulation was < $2 \mu \mathrm{m}$. In addition, we selected a presynaptic varicosity which was apparently distant from neighbor varicosities by $>2 \mu \mathrm{m}$, so that only one presynaptic varicosity would be activated by the electrical stimulation. 


\section{Quantal EPSCs on hippocampal pyramidal neurons}

Single synaptic responses were noisy and variable in some cases. Therefore, we wished to use an objective method to analyze them. To this end, we adopted the following wave-form classification-based method. All EPSCs recorded from a neuron in a particular stimulation condition were classified by the similarity of their amplitudes and time courses (Fig. 3A). This classification was performed by cluster analysis with scores reflecting differences of EPSC wave-forms (for details, see Methods). Then, the averaged EPSC traces of the respective groups were calculated, and the quantal content for each group was determined (Fig. 3B). Finally, the qEPSC trace was obtained by averaging EPSCs divided by the respective quantal content (Fig. 3C). The quantal amplitude of EPSCs shown in Fig. 1 estimated by this method was 5.5 pA, which was close to the amplitude of the smallest peak in the amplitude histogram (6 pA, Fig. 1E). The amplitude, the 10\% - 90\% rise time and the 10\% - 90\% decay time of qEPSCs, and the mean quantal contents (excluding failure events) were measured as physiological properties of EPSCs at hippocampal synapses. The means \pm S.D. of these values are presented in Table 1. 


\section{Granule neuron - Purkinje neuron synapses}

We next examined the properties of granule neuron - Purkinje neuron synapses in a cerebellar dissociated culture preparation. Essentially similar methods as those used in hippocampal cultures were used. In some experiments, we labeled Purkinje neurons with EGFP using AAV and presynaptic neurons with turboRFP. This procedure enabled us to visualize synaptic contacts before performing whole-cell patch-clamp recording, and facilitated finding isolated synapses. Representative data recorded from a Purkinje neuron are shown in Fig 4. We recorded EPSCs with similar time courses and also failure events by stimulating a presynaptic varicosity on the Purkinje neuron (Fig. 4D). The histogram of EPSC amplitudes showed three peaks (10 pA, 20 pA and 30 pA; Fig 4E). Quantal EPSC was estimated by the wave-form classification-based method (Fig. $4 \mathrm{~F})$. The estimated amplitude of qEPSC was $9.7 \mathrm{pA}$, which was similar to the smallest peak value of evoked EPSCs in the amplitude histogram. The amplitude, the $10 \%-90 \%$ rise time and the $10 \%-90 \%$ decay time of qEPSCs, and the mean quantal number (excluding failure events) were measured as physiological properties of EPSCs at cerebellar granule neuron - Purkinje neuron synapses. The means \pm S.D. of these values are presented in Table 1. An FM1-43 imaging experiment was also performed in the cerebellar culture. The effective area of electrical stimulation was limited to $<2 \mu \mathrm{m}$ 
from the center of the electrode tip, as in the hippocampal culture (Fig. 5). The intensity of electrical stimulation used for EPSC recordings in the cerebellar culture was $10-20$ $\mathrm{V}$ in most cases. We also selected a presynaptic varicosity distant from neighbor varicosities by $>2 \mu \mathrm{m}$, so that only one presynaptic varicosity would be stimulated.

Comparison of physiological and morphological properties between hippocampal and cerebellar synapses

Physiological and morphological properties of the hippocampal and cerebellar synapses are presented in Table 1 . The time courses of qEPSCs at hippocampal synapses were faster than those at cerebellar synapses. Both the $10 \%-90 \%$ rise and the $10 \%-90 \%$ decay were significantly faster at the hippocampal synapses than at the cerebellar synapses. The mean quantal numbers of EPSCs were significantly smaller in the hippocampus. In contrast, no significant difference was detected in the quantal amplitudes.

As morphological properties, the sizes of presynaptic varicosities and of apposed postsynaptic spines were measured. The presynaptic varicosity boundary was outlined by thresholding a z-stacked image. On the other hand, the spine area was determined by finding a signal intensity valley (for details, see Methods). This method was adopted 
because the fluorescent signal was much higher in dendritic shafts than in spines (particularly in Purkinje neurons), which made the simple thresholding method unreliable for determining spine boundaries. Postsynaptic spine sizes were found here to be larger in Purkinje neurons than in hippocampal neurons, as reported previously (Harris and Stevens, 1989), whereas no significant difference was detected in presynaptic varicosity sizes.

Correlations among properties of quantal EPSCs and synaptic morphology

Next, we analyzed the correlations among properties of synaptic morphology and EPSCs. In addition to the properties shown in Table 1, we measured the distance between a stimulated synapse and the soma, and analyzed correlations among these properties at glutamatergic synapses on hippocampal pyramidal neurons and glutamatergic synapses on cerebellar Purkinje neurons. The correlations at hippocampal synapses are plotted and regression lines are shown in Fig. 6, while Fig. 7 shows the corresponding data in the cerebellum. Pearson product-moment correlation coefficients are also shown.

In the hippocampus, the postsynaptic spine size and the amplitude of qEPSC showed a statistically significant positive correlation. The $10-90 \%$ decay time was also 
negatively correlated with the size of the postsynaptic spine in the hippocampus. In contrast, such correlations were not detected in the cerebellum. The size of the presynaptic varicosity was correlated with the postsynaptic spine size and also with the mean quantal number of EPSCs only in the cerebellum. In this study, we failed to detect any correlation between the location of a synapse and other properties in both the hippocampus and the cerebellum. This might have been partly because of the limited variation of sampling locations of synapses within neurons.

\section{Discussion}

We have established a method to analyze single synaptic responses together with the pre- and postsynaptic morphology. A presynaptic axon and a postsynaptic neuron forming a synapse were labeled with different fluorescent dyes, and a presumptive presynaptic varicosity was stimulated under the suppression of action potential. Glutamatergic synapses on hippocampal pyramidal neurons and on cerebellar Purkinje neurons were analyzed in primary cultures. Some properties of these two types of synapse were different. The sizes of postsynaptic spines in the hippocampus were smaller than those in the cerebellum, and the time courses of qEPSCs at hippocampal synapses were faster than those at cerebellar synapses. Some correlations among 
morphological and qEPSC properties were also different. The postsynaptic spine size was positively correlated with the qEPSC amplitude and negatively correlated with the $10-90 \%$ decay time of qEPSC only at the hippocampal synapses. In contrast, the size of the presynaptic varicosity was positively correlated with the size of the postsynaptic spine and the mean quantal number of EPSCs only at the cerebellar synapses.

\section{Single synapse stimulation}

Minimum electrical stimulation has been used to activate one or two synapses (Allen and Stevens, 1994; Denk et al., 1995; Dobrunz and Stevens, 1997). In subsequent studies, single synaptic responses were recorded by applying minimum stimulation to the FM-dye-labeled structures in the presence of TTX (Chen et al., 2004; Fedulova et al., 1999; Kirischuk et al., 1999). We have adopted a similar approach, and extended it so that both presynaptic and postsynaptic structures could be observed in detail using the fluorescent protein EGFP and the fluorescent protein turboRFP or fluorescent dye Alexa594. This method enabled us to record single synaptic responses together with the morphology of both a presynaptic varicosity and a postsynaptic spine.

In this study, we estimated the effective stimulation area with FM 1-43 dye: it was within $<2 \mu \mathrm{m}$ from the center of the stimulation electrode tip. This spread of the 
stimulation was similar to or smaller than those seen in previous studies using electrical stimulation in the presence of TTX (Chen et al., 2004; Fedulova et al., 1999; Kirischuk et al., 1999) or those using local application of hypertonic or high $\mathrm{K}^{+}$solutions (Bekkers et al., 1990; Liu and Tsien, 1995; McAllister and Stevens, 2000; Murnick et al., 2002; Stevens and Tsujimoto, 1995). In the present study, we selected a varicosity which was apparently distant from neighbors by $>2 \mu \mathrm{m}$. Further, data were discarded when evoked EPSCs showed multiple time courses. Thus, we think that most, if not all, of the analyzed EPSCs were evoked by activation of a single presynaptic varicosity. One problem of this method is the difficulty of maintaining stable recording of qEPSCs for a long time. Under the suppression of action potential generation with TTX, strong electrical stimulation might be required to depolarize a presynaptic varicosity sufficiently to induce the release of synaptic vesicles, and such strong electrical stimulations, when repeated, might cause some damage to the presynaptic varicosity.

\section{Quantal EPSC estimation and quantal content}

In this study, qEPSCs were estimated using a wave-form classification-based method. A histogram-based method has been widely used to estimate quantal amplitude (Foster and McNaughton, 1991; Kullmann and Nicoll, 1992; Liao et al., 1992; Stricker et al., 
1996), in which only the peak amplitude is used and the time-course information is discarded. In contrast, both of them are used in a wave-form classification-based method, and EPSCs with different time courses could be objectively classified into different groups.

In the central nervous system, the one presynaptic varicosity - one vesicle hypothesis gained wide acceptance (Redman, 1990). However, recent studies revealed that multivesicular releases occur from a presynaptic varicosity, and single vesicular release does not necessarily saturate postsynaptic responses (Christie and Jahr, 2006; Conti and Lisman, 2003; Foster et al., 2005; Liu, 2003; Tong and Jahr, 1994). The present results are in line with the latter idea that multivesicular releases occur at both hippocampal and cerebellar synapses. It has been reported that around 10 vesicles are docked at a presynaptic active zone of a hippocampal CA1 neuron (Schikorski and Stevens, 1997), and 8 vesicles are docked at each active zone of a parallel fiber (axon of granule neuron) varicosity (Xu-Friedman et al., 2001). Thus, multivesicular releases might occur from a single active zone. In the hippocampus, $90 \%$ of presynaptic varicosities have only one synaptic contact in vivo (Schikorski and Stevens, 1997). On the other hand, $27 \%$ of parallel fiber varicosities have more than one synaptic contact with spines of a Purkinje neuron in vivo (Harris and Stevens, 1988). Thus, synaptic 
vesicles might be released from multiple active zones of a presynaptic varicosity of a granule neuron. This might have contributed to the difference in quantal contents between the two types of synapses observed in this study, although EPSCs induced by the transmitter released from different active zones might be classified into different groups and might not have been analyzed in this study. A large number of failure events and significant numbers of EPSCs with multiple quanta followed the electrical stimulations with threshold intensity. The distribution of quantal contents of EPSCs could not be fitted by a binominal distribution with a fixed release probability of synaptic vesicles. This result was unexpected. The electrical pulse to depolarize a presynaptic varicosity in the presence of TTX might cause variable increases in the intracellular $\mathrm{Ca}^{++}$increases depending on the condition of the varicosity, as generation of an all-or-none action potential was suppressed. Such variation in the presynaptic $\mathrm{Ca}^{++}$response might have caused unstability in the release probability. Alternatively, there might be a mechanism to couple release of synaptic vesicles in the presynaptic varicosities.

We also found that the time courses of qEPSCs in the hippocampus were faster than those in the cerebellum, which is consistent with previous reports (Bekkers et al., 1990; Belmeguenai et al., 2008; Hirano and Hagiwara, 1988; Stevens and Williams, 2007). 
The cause of this is unclear. It might reflect differences of AMPAR subunit compositions and/or dendritic filtering effects in the two types of neurons (Martin et al., 1993;

Spruston et al., 1994)

Correlations among physiological and morphological properties

We found that larger qEPSCs were induced at larger postsynaptic spines in the hippocampus, where both the efficacy of synaptic transmission and the postsynaptic spine structure are likely determined by neuronal activities. It is known that LTP is accompanied by spine enlargement (Matsuzaki et al., 2004; Okamoto et al., 2004) and LTD by spine shrinkage (Okamoto et al., 2004; Zhou et al., 2004). Therefore, spontaneously occurring LTP and LTD in the hippocampal culture might have caused the positive correlation between qEPSC amplitude and the size of postsynaptic spine. We also noticed a tendency for slowly decaying qEPSCs to be recorded at small postsynaptic spines. How this correlation occurred is not known. Substitution of AMPAR with different subunit compositions occurs in hippocampal LTP (Shi et al., 2001), which might change the time course of the EPSC at a single synapse. Hippocampal pyramidal neurons express not only AMPARs but also NMDARs, which show slower kinetics. The increase in the relative amount of AMPAR to NMDAR 
component caused by LTP might have decreased the slow component of qEPSC and the $10-90 \%$ decay time. However, we think this is unlikely, because all experiments were performed in $\mathrm{Mg}^{++-}$containing external solution, which should have strongly blocked NMDAR-mediated current.

In contrast, at cerebellar granule neurons - Purkinje neuron synapses, neither the amplitude nor the time course of qEPSC was correlated with the size of postsynaptic spine. At this type of synapse, NMDARs are not expressed (Hirano and Hagiwara, 1988), and LTD occurs without a decrease in postsynaptic spine volume (Sdrulla and Linden, 2007). Larger presynaptic varicosities of granule neurons tended to form synapses with larger postsynaptic spines and release more synaptic vesicles. Presynaptic varicosity size might be related to the release probability of synaptic vesicles in granule neurons. It was previously reported that there are positive morphological correlation between presynaptic varicosities of parallel-fiber and postsynaptic spine of Purkinje neurons. However, such correlations were not found between presynaptic varicosities of ascending granule cell axon and postsynaptic spines (Gundappa-Sulur et al., 1999). Thus, the correlation we detected in the culture might reflect properties of parallel-fiber type synapses, which are abundant in vivo. The morphological correlation between presynaptic varicosities and postsynaptic spines suggests that there may be a 
mechanism to coordinate the pre- and postsynaptic elements at this type of synapses.

Selective expression of glutamate receptor-like molecule GluR82 in Purkinje neurons might contribute to the coordination. In GluR82 knockout mice, the number of granule neuron - Purkinje neuron synapses is reduced, and a mismatch occurs between presynaptic active zones and the postsynaptic densities (Kashiwabuchi et al., 1995; Takeuchi et al., 2005). In addition, the direct synaptogenic ability of GluR82 was reported (Kuroyanagi et al., 2009). We did not detect a significant correlation between the sizes of presynaptic varicosities and postsynaptic spines at the hippocampal synapses, although strong matching of presynaptic active zones and postsynaptic densities was reported (Schikorski and Stevens, 1997). Sizes of presynaptic varicosity and postsynaptic spines might not necessarily be correlated with sizes of active zones and postsynaptic densities, respectively. The present results suggest that the regulatory mechanisms of synaptic morphology and function are different between hippocampal and cerebellar synapses. 


\section{Acknowledgements}

This work was supported by a grant from the Ministry of Education, Culture, Sports,

Science, and Technology, Japan (MEXT) to T.H., and by Global COE program A06 to

Kyoto University. We thank Drs. E. Nakajima, Y. Tagawa and S. Kawaguchi for helpful comments on the manuscript. 


\section{References}

Allen C, Stevens CF. 1994. An evaluation of causes for unreliability of synaptic transmission. Proc Natl Acad Sci U S A 91(22):10380-10383.

Andrasfalvy BK, Magee JC. 2001. Distance-dependent increase in AMPA receptor number in the dendrites of adult hippocampal CA1 pyramidal neurons. J Neurosci 21(23):9151-9159.

Bekkers JM, Richerson GB, Stevens CF. 1990. Origin of variability in quantal size in cultured hippocampal neurons and hippocampal slices. Proc Natl Acad Sci U S A 87(14):5359-5362.

Belmeguenai A, Botta P, Weber JT, Carta M, De Ruiter M, De Zeeuw CI, Valenzuela CF, Hansel C. 2008. Alcohol impairs long-term depression at the cerebellar parallel fiber-Purkinje cell synapse. J Neurophysiol 100(6):3167-3174.

Brewer GJ, Torricelli JR, Evege EK, Price PJ. 1993. Optimized survival of hippocampal neurons in B27-supplemented Neurobasal, a new serum-free medium combination. J Neurosci Res 35(5):567-576.

Chen G, Harata NC, Tsien RW. 2004. Paired-pulse depression of unitary quantal amplitude at single hippocampal synapses. Proc Natl Acad Sci U S A 101(4):1063-1068.

Christie JM, Jahr CE. 2006. Multivesicular release at Schaffer collateral-CA1 hippocampal synapses. J Neurosci 26(1):210-216.

Conti R, Lisman J. 2003. The high variance of AMPA receptor- and NMDA receptor-mediated responses at single hippocampal synapses: evidence for multiquantal release. Proc Natl Acad Sci U S A 100(8):4885-4890.

Denk W, Sugimori M, Llinas R. 1995. Two types of calcium response limited to single spines in cerebellar Purkinje cells. Proc Natl Acad Sci U S A 92(18):8279-8282.

Dobrunz LE, Stevens CF. 1997. Heterogeneity of release probability, facilitation, and depletion at central synapses. Neuron 18(6):995-1008.

Fedulova SA, Vasilyev DV, Isaeva EV, Romanyuk SG, Veselovsky NS. 1999. Possibility of multiquantal transmission at single inhibitory synapse in cultured rat hippocampal neurons. Neuroscience 92(4):1217-1230.

Foster KA, Crowley JJ, Regehr WG. 2005. The influence of multivesicular release and postsynaptic receptor saturation on transmission at granule cell to Purkinje cell synapses. J Neurosci 25(50):11655-11665.

Foster TC, McNaughton BL. 1991. Long-term enhancement of CA1 synaptic transmission is due to increased quantal size, not quantal content. Hippocampus 1(1):79-91.

Furuya S, Makino A, Hirabayashi Y. 1998. An improved method for culturing cerebellar 
Purkinje cells with differentiated dendrites under a mixed monolayer setting. Brain Res Brain Res Protoc 3(2):192-198.

Futai K, Kim MJ, Hashikawa T, Scheiffele P, Sheng M, Hayashi Y. 2007. Retrograde modulation of presynaptic release probability through signaling mediated by PSD-95-neuroligin. Nat Neurosci 10(2):186-195.

Gundappa-Sulur G, De Schutter E, Bower JM. 1999. Ascending granule cell axon: an important component of cerebellar cortical circuitry. J Comp Neurol 408(4):580-596.

Harris KM, Landis DM. 1986. Membrane structure at synaptic junctions in area CA1 of the rat hippocampus. Neuroscience 19(3):857-872.

Harris KM, Stevens JK. 1988. Dendritic spines of rat cerebellar Purkinje cells: serial electron microscopy with reference to their biophysical characteristics. J Neurosci 8(12):4455-4469.

Harris KM, Stevens JK. 1989. Dendritic spines of CA 1 pyramidal cells in the rat hippocampus: serial electron microscopy with reference to their biophysical characteristics. J Neurosci 9(8):2982-2997.

Hirano T, Hagiwara S. 1988. Synaptic transmission between rat cerebellar granule and Purkinje cells in dissociated cell culture: effects of excitatory-amino acid transmitter antagonists. Proc Natl Acad Sci U S A 85(3):934-938.

Hirano T, Kasono K. 1993. Spatial distribution of excitatory and inhibitory synapses on a Purkinje cell in a rat cerebellar culture. J Neurophysiol 70(4):1316-1325.

Horikawa K, Takeichi M. 2001. Requirement of the juxtamembrane domain of the cadherin cytoplasmic tail for morphogenetic cell rearrangement during myotome development. J Cell Biol 155(7):1297-1306.

Kashiwabuchi N, Ikeda K, Araki K, Hirano T, Shibuki K, Takayama C, Inoue Y, Kutsuwada T, Yagi T, Kang Y, Aizawa S, Mishina M. 1995. Impairment of motor coordination, Purkinje cell synapse formation, and cerebellar long-term depression in GluRס2 mutant mice. Cell 81(2):245-252.

Kawaguchi SY, Hirano T. 2006. Integrin a381 suppresses long-term potentiation at inhibitory synapses on the cerebellar Purkinje neuron. Mol Cell Neurosci 31(3):416-426.

Kirischuk S, Veselovsky N, Grantyn R. 1999. Relationship between presynaptic calcium transients and postsynaptic currents at single gamma-aminobutyric acid (GABA)ergic boutons. Proc Natl Acad Sci U S A 96(13):7520-7525.

Kullmann DM, Nicoll RA. 1992. Long-term potentiation is associated with increases in quantal content and quantal amplitude. Nature 357(6375):240-244.

Kuroyanagi T, Yokoyama M, Hirano T. 2009. Postsynaptic glutamate receptor $\delta$ family 
contributes to presynaptic terminal differentiation and establishment of synaptic transmission. Proc Natl Acad Sci U S A 106(12):4912-4916.

Lee KJ, Kim H, Kim TS, Park SH, Rhyu IJ. 2004. Morphological analysis of spine shapes of Purkinje cell dendrites in the rat cerebellum using high-voltage electron microscopy. Neurosci Lett 359(1-2):21-24.

Liao D, Jones A, Malinow R. 1992. Direct measurement of quantal changes underlying long-term potentiation in CA1 hippocampus. Neuron 9(6):1089-1097.

Liu G. 2003. Presynaptic control of quantal size: kinetic mechanisms and implications for synaptic transmission and plasticity. Curr Opin Neurobiol 13(3):324-331.

Liu G, Tsien RW. 1995. Properties of synaptic transmission at single hippocampal synaptic boutons. Nature 375(6530):404-408.

Magee JC, Cook EP. 2000. Somatic EPSP amplitude is independent of synapse location in hippocampal pyramidal neurons. Nat Neurosci 3(9):895-903.

Martin LJ, Blackstone CD, Levey AI, Huganir RL, Price DL. 1993. AMPA glutamate receptor subunits are differentially distributed in rat brain. Neuroscience 53(2):327-358.

Matsuzaki M, Honkura N, Ellis-Davies GC, Kasai H. 2004. Structural basis of long-term potentiation in single dendritic spines. Nature 429(6993):761-766.

McAllister AK, Stevens CF. 2000. Nonsaturation of AMPA and NMDA receptors at hippocampal synapses. Proc Natl Acad Sci U S A 97(11):6173-6178.

Mizuno H, Hirano T, Tagawa Y. 2007. Evidence for activity-dependent cortical wiring: formation of interhemispheric connections in neonatal mouse visual cortex requires projection neuron activity. J Neurosci 27(25):6760-6770.

Mizuno H, Hirano T, Tagawa Y. 2010. Pre- and postsynaptic neuron activity supports the axon development of callosal projection neurons during different postnatal periods in the cerebral cortex. Eur J Neurosci 31(3):410-424.

Murnick JG, Dube G, Krupa B, Liu G. 2002. High-resolution iontophoresis for single-synapse stimulation. J Neurosci Methods 116(1):65-75.

Murphy TH, Baraban JM, Wier WG. 1995. Mapping miniature synaptic currents to single synapses using calcium imaging reveals heterogeneity in postsynaptic output. Neuron 15(1):159-168.

Murthy VN, Sejnowski TJ, Stevens CF. 1997. Heterogeneous release properties of visualized individual hippocampal synapses. Neuron 18(4):599-612.

Nicholson DA, Trana R, Katz Y, Kath WL, Spruston N, Geinisman Y. 2006. Distance-dependent differences in synapse number and AMPA receptor expression in hippocampal CA1 pyramidal neurons. Neuron 50(3):431-442. 
Nusser Z, Cull-Candy S, Farrant M. 1997. Differences in synaptic GABA(A) receptor number underlie variation in GABA mini amplitude. Neuron 19(3):697-709.

Nusser Z, Lujan R, Laube G, Roberts JD, Molnar E, Somogyi P. 1998. Cell type and pathway dependence of synaptic AMPA receptor number and variability in the hippocampus. Neuron 21(3):545-559.

Okamoto K, Nagai T, Miyawaki A, Hayashi Y. 2004. Rapid and persistent modulation of actin dynamics regulates postsynaptic reorganization underlying bidirectional plasticity. Nat Neurosci 7(10):1104-1112.

Redman S. 1990. Quantal analysis of synaptic potentials in neurons of the central nervous system. Physiol Rev 70(1):165-198.

Schikorski T, Stevens CF. 1997. Quantitative ultrastructural analysis of hippocampal excitatory synapses. J Neurosci 17(15):5858-5867.

Sdrulla AD, Linden DJ. 2007. Double dissociation between long-term depression and dendritic spine morphology in cerebellar Purkinje cells. Nat Neurosci 10(5):546-548.

Shi S, Hayashi Y, Esteban JA, Malinow R. 2001. Subunit-specific rules governing AMPA receptor trafficking to synapses in hippocampal pyramidal neurons. Cell 105(3):331-343.

Smith MA, Ellis-Davies GC, Magee JC. 2003. Mechanism of the distance-dependent scaling of Schaffer collateral synapses in rat CA1 pyramidal neurons. J Physiol 548(Pt 1):245-258.

Spruston N, Jaffe DB, Johnston D. 1994. Dendritic attenuation of synaptic potentials and currents: the role of passive membrane properties. Trends Neurosci 17(4):161-166.

Stevens CF, Tsujimoto T. 1995. Estimates for the pool size of releasable quanta at a single central synapse and for the time required to refill the pool. Proc Natl Acad Sci U S A $92(3): 846-849$.

Stevens CF, Williams JH. 2000. "Kiss and run" exocytosis at hippocampal synapses. Proc Natl Acad Sci U S A 97(23):12828-12833.

Stevens CF, Williams JH. 2007. Discharge of the readily releasable pool with action potentials at hippocampal synapses. J Neurophysiol 98(6):3221-3229.

Stricker C, Field AC, Redman SJ. 1996. Statistical analysis of amplitude fluctuations in EPSCs evoked in rat CA1 pyramidal neurones in vitro. J Physiol 490 ( Pt 2):419-441.

Takeuchi T, Miyazaki T, Watanabe M, Mori H, Sakimura K, Mishina M. 2005. Control of synaptic connection by glutamate receptor $\delta 2$ in the adult cerebellum. J Neurosci 25(8):2146-2156.

Tanaka K, Khiroug L, Santamaria F, Doi T, Ogasawara H, Ellis-Davies GC, Kawato M, Augustine GJ. 2007. $\mathrm{Ca}^{2+}$ requirements for cerebellar long-term synaptic depression: 
role for a postsynaptic leaky integrator. Neuron 54(5):787-800.

Tong G, Jahr CE. 1994. Multivesicular release from excitatory synapses of cultured hippocampal neurons. Neuron 12(1):51-59.

Ward JH. 1963. Hierarchical Grouping to Optimize an Objective Function. Journal of the American Statistical Association 58(301):236-244.

Xu-Friedman MA, Harris KM, Regehr WG. 2001. Three-dimensional comparison of ultrastructural characteristics at depressing and facilitating synapses onto cerebellar Purkinje cells. J Neurosci 21(17):6666-6672.

Zhou Q, Homma KJ, Poo MM. 2004. Shrinkage of dendritic spines associated with long-term depression of hippocampal synapses. Neuron 44(5):749-757. 
Table 1. Physiological and morphological properties

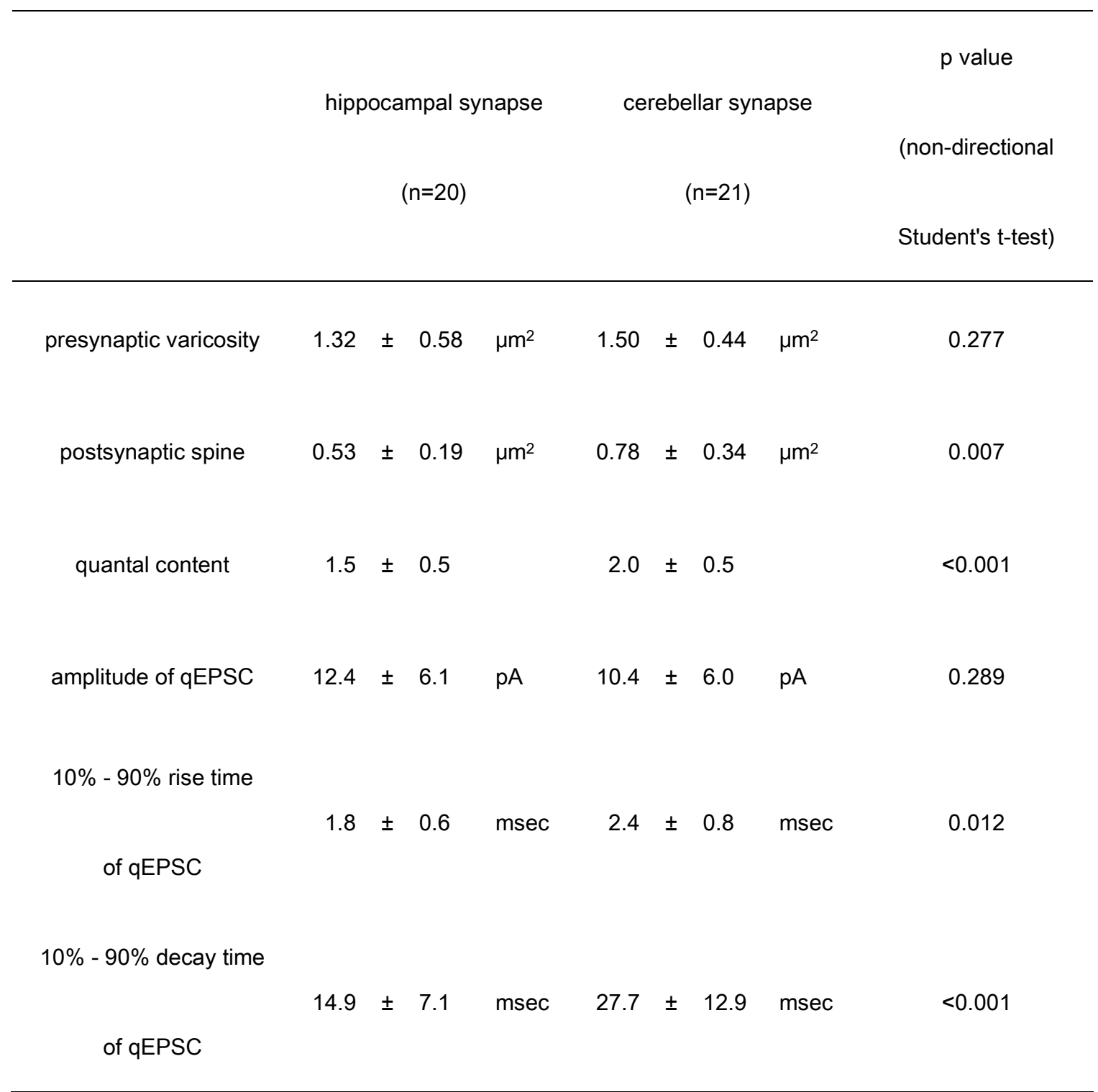

Values are means \pm S.D. 
Figure legends

Figure 1

Single synapse on a hippocampal pyramidal neuron. A,B; A hippocampal pyramidal neuron (magenta, an arrow indicates the soma) and axons and dendrites of other hippocampal neurons (green) are shown. The stimulated synapse was located in the upper center position surrounded by a white square, and higher magnification views are shown in B. In A 17 images at different focal planes were stacked and 8 images were stacked in B. The stimulation glass electrode is drawn with white lines in B c. C; 3D reconstructed image of the synapse. D; Ten representative EPSC traces evoked by stimulation with the electrode drawn in B. E; Histogram of evoked EPSC amplitudes.

Figure 2

Estimation of the spatial spread of electrical stimulation in the hippocampal culture. A; Presynaptic terminals were loaded with FM1-43 (magenta). Phase contrast images with FM1-43 fluorescent signals are shown. a, before electrical stimulation, b, after electrical stimulation (30V, $10 \mathrm{~Hz}, 10$ seconds), c, after application of a high $\mathrm{K}^{+}$solution. High $\mathrm{K}^{+}$ solution was applied to confirm that most FM 1-43 signals would be unloaded by 
depolarization of all varicosities. Green circles indicate $2 \mu \mathrm{m}, 5 \mu \mathrm{m}$, and $10 \mu \mathrm{m}$ distances from the center of the stimulation electrode tip. B; The time courses of fluorescent intensity before and after the electrical and high $\mathrm{K}^{+}$stimulations. Averaged signal intensities of FM1-43-positive areas were measured. Means \pm S.E.M.s $(n=7)$ of the averaged intensity for each area are shown.

Figure 3

Estimation of qEPSC. A; EPSCs evoked at the synapse shown in Figure1 were classified into three groups $(0,1,2)$. In each panel, $6-13$ traces are superimposed. B; EPSCs belonging to each group shown in A were averaged. The quantal number (in parentheses) of each group was estimated based on these traces. C; The qEPSC estimated by averaging EPSC traces divided by the respective quantal numbers.

\section{Figure 4}

Single cerebellar granule neuron - Purkinje neuron synapse. A,B; A Purkinje neuron (magenta, the arrow indicates the soma) and axons and dendrites of granule neurons (green) are shown. The stimulated synapse was located in the upper center position surrounded by a white square, and higher magnification views are presented in B. In A 
13 images at different focal planes were stacked and 8 images were stacked in B. The stimulation glass electrode is drawn with white lines in B c. C; 3D reconstructed image of the synapse. D; Ten representative EPSC traces evoked with the stimulation glass electrode drawn in B. E; Histogram of evoked EPSC amplitudes. F; Estimated qEPSC at the synapse.

Figure 5

Estimation of the spatial spread of electrical stimulation in the cerebellar culture. A; Presynaptic terminals were loaded with FM1-43 (magenta). Phase contrast images with FM1-43 fluorescent signals are shown. a, before electrical stimulation, b, after electrical stimulation (30V, $10 \mathrm{~Hz}, 10$ seconds), c, after application of a high $\mathrm{K}^{+}$solution. Green circles indicate $2 \mu \mathrm{m}, 5 \mu \mathrm{m}$, and $10 \mu \mathrm{m}$ distances from the center of the stimulation electrode tip. B; The time courses of fluorescent intensity before and after the electrical and high $\mathrm{K}^{+}$stimulations. Averaged signal intensities of FM1-43-positive areas were measured. Means \pm S.E.M.s $(n=7)$ of the averaged intensity for each are shown.

Figure 6

Scatter plots and statistical values of physiological and morphological properties at 
hippocampal synapses. Lower left part: Scatter plots of measured values. Pairs with statistically significant correlations are plotted with filled circles, otherwise with open circles. Solid lines are the linear regression lines. Diagonal part: Histograms of measured values. Upper right part: Statistical data. Pearson's product-moment correlation coefficients $(r)$ and $\mathrm{t}^{-}$and $\mathrm{p}^{-v}$ values of the test for significance of the correlation coefficient $(t, P)$, are shown.

Figure 7

Scatter plots and statistical values of physiological and morphological properties at cerebellar synapses. Lower left part: Scatter plots of measured values. Pairs with statistically significant correlations are plotted with filled circles and otherwise with open circles. Solid lines indicate the linear regression lines. Diagonal part: Histograms of measured values. Upper right part: Statistical data. Pearson's product-moment correlation coefficients $(r)$ and $\mathrm{p}$ values of the test for significance of the correlation coefficient $(P)$ are shown. 

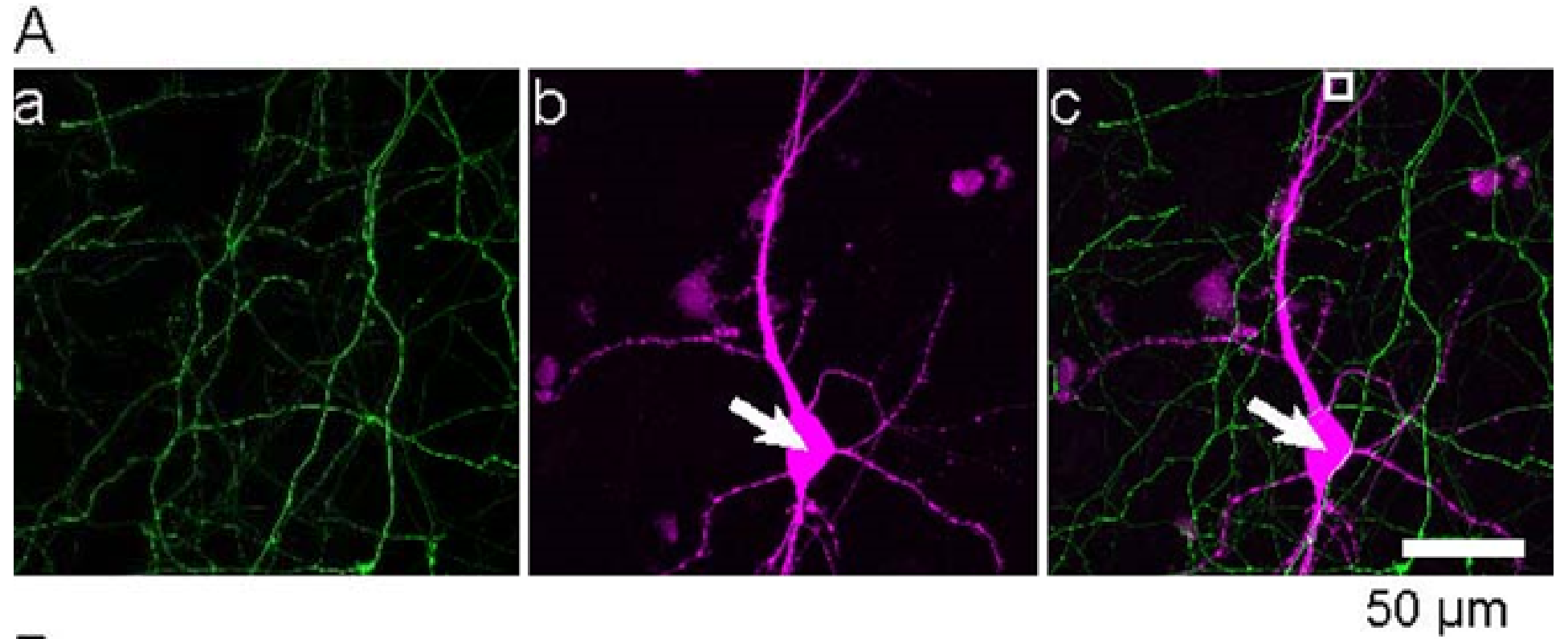

\section{B}
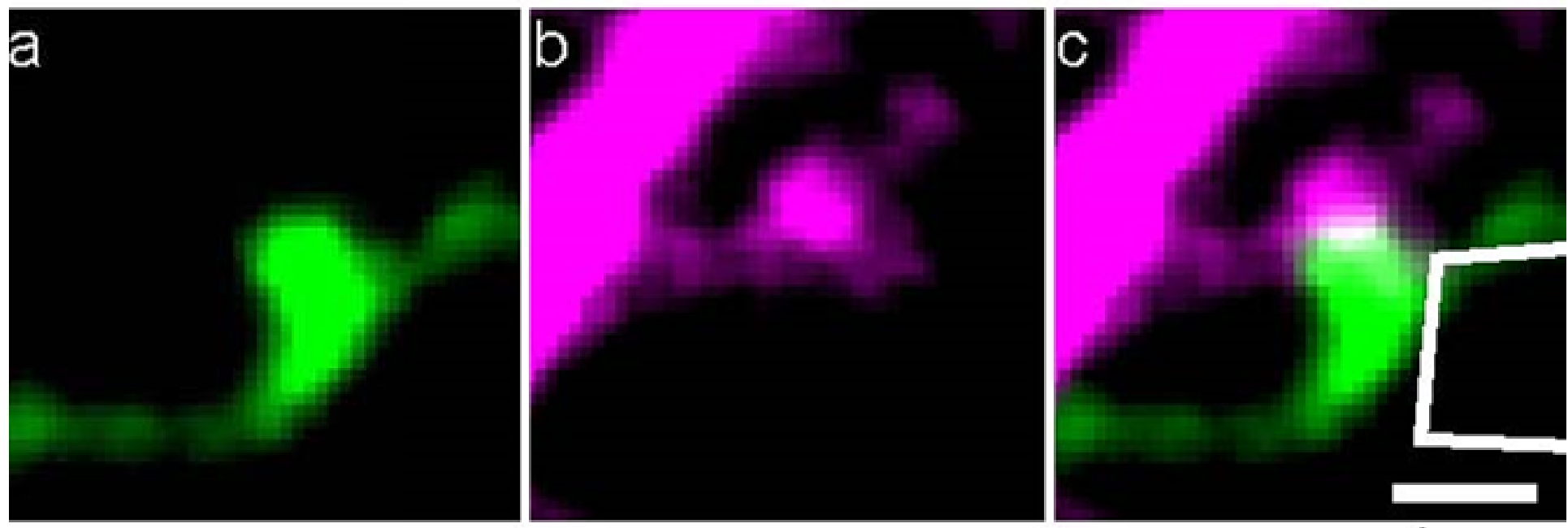

$1 \mu \mathrm{m}$

C

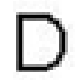

E
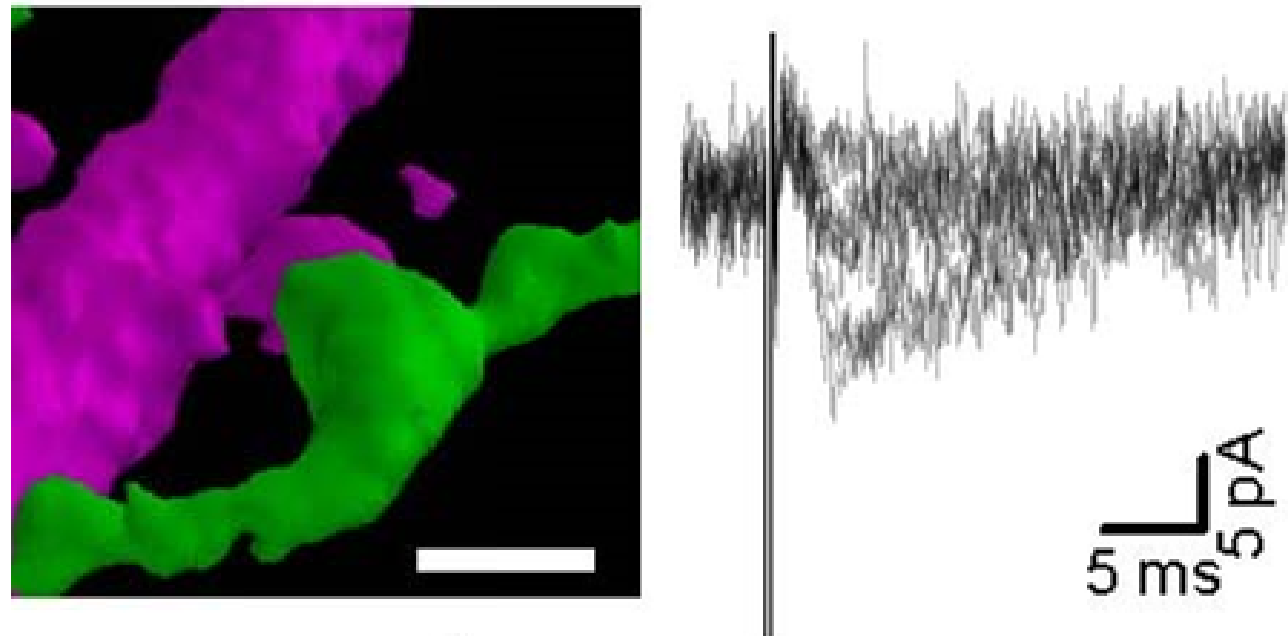

$1 \mu \mathrm{m}$

Figure 1 
A
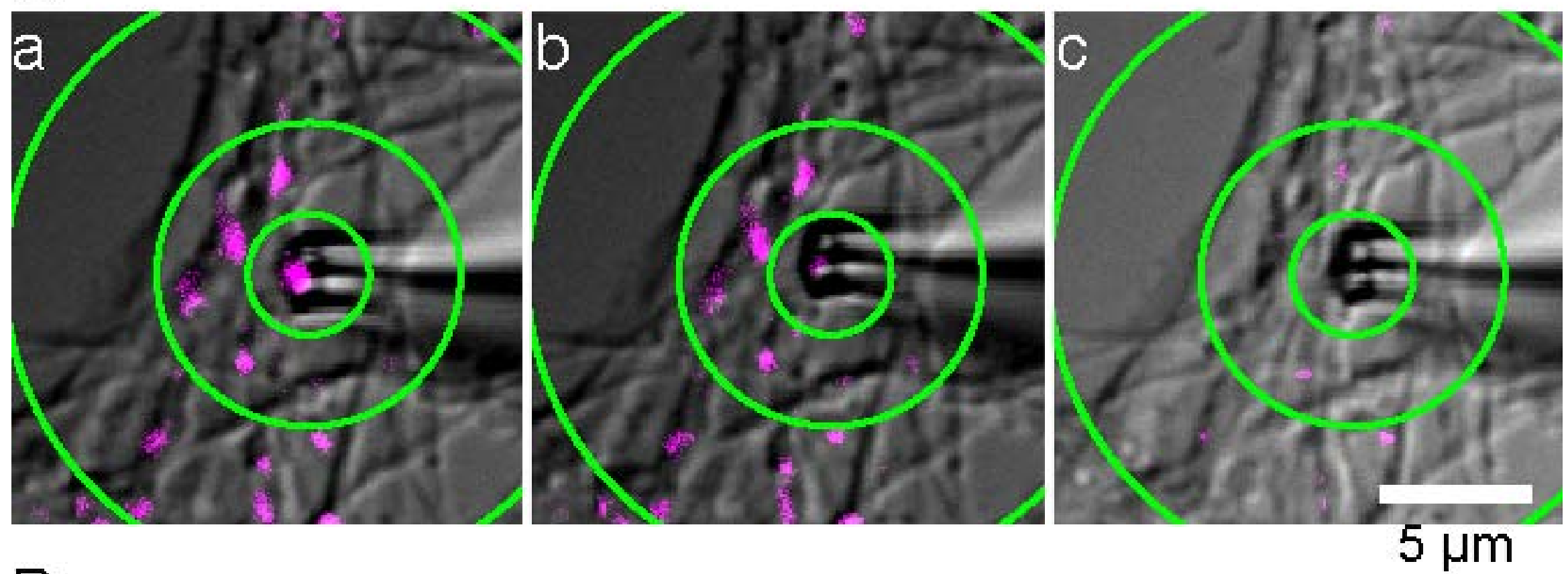

B

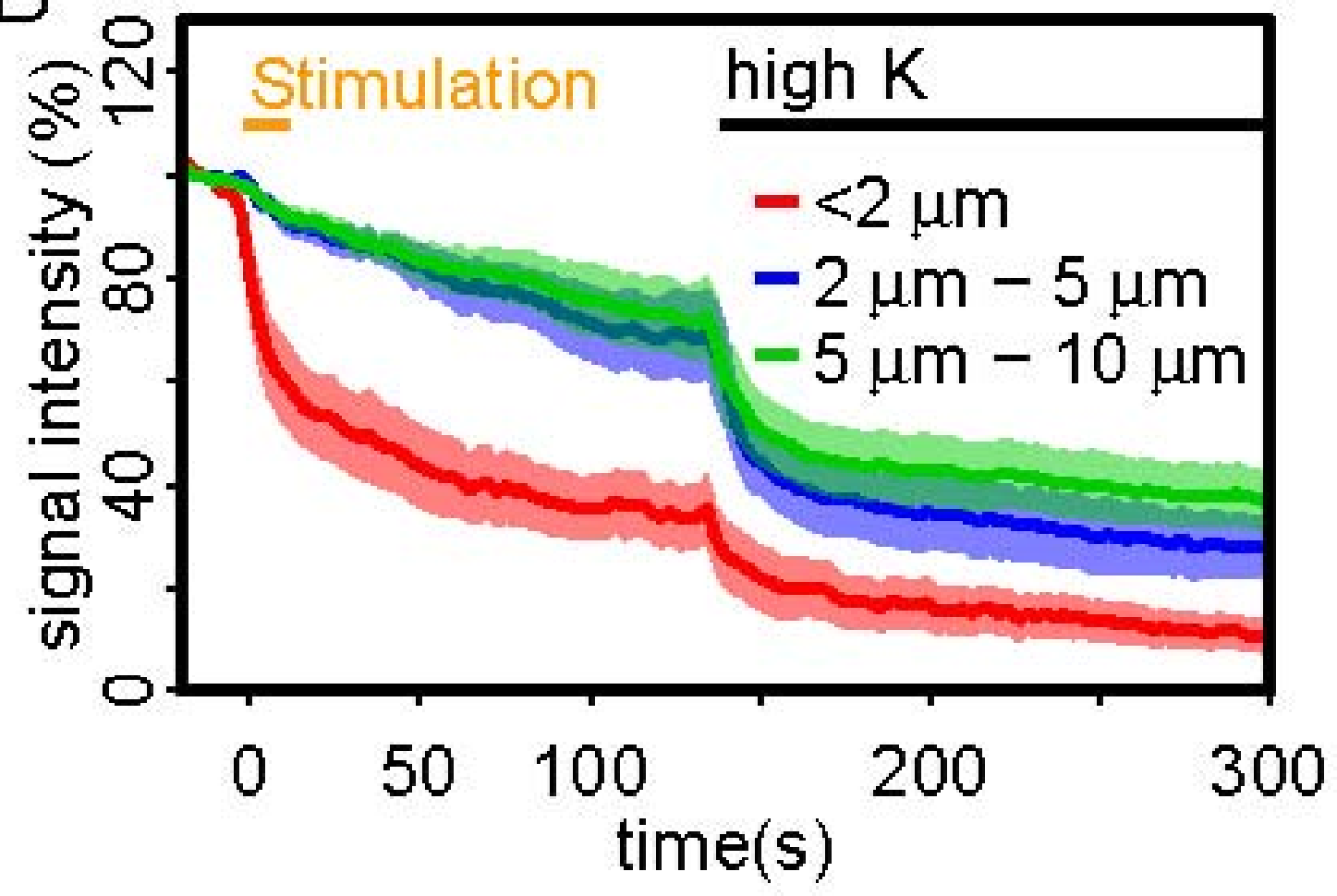

Figure 2 
A

(0)

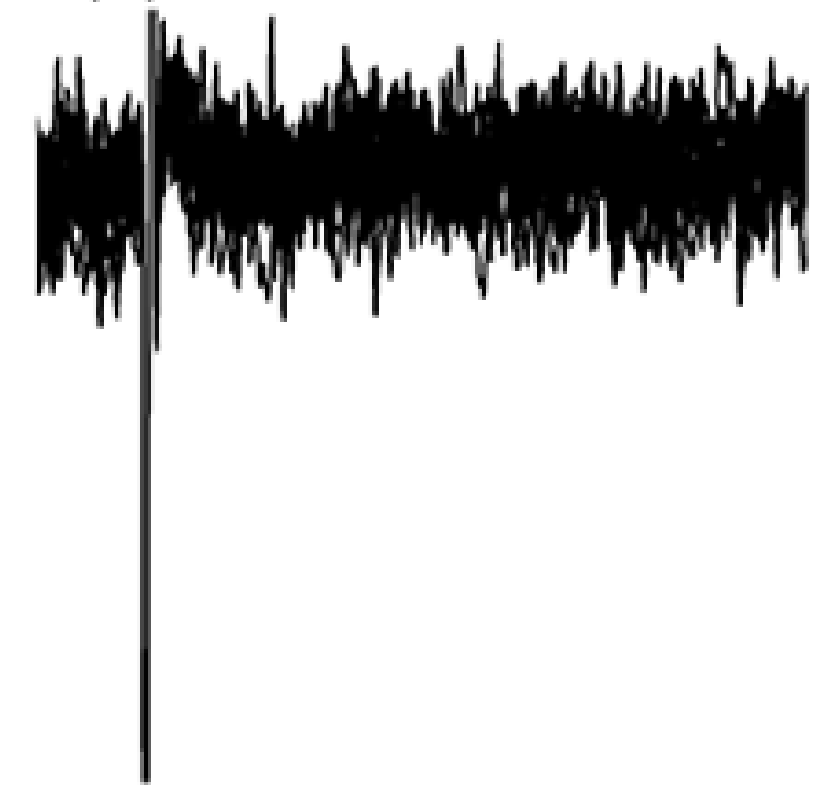

B
(1)

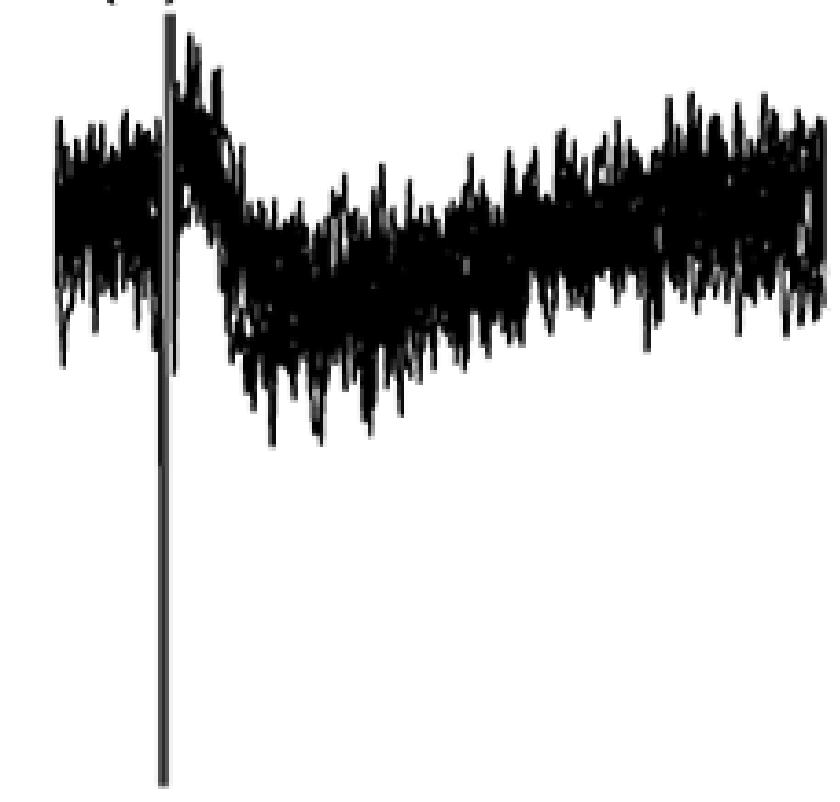

C
(2)

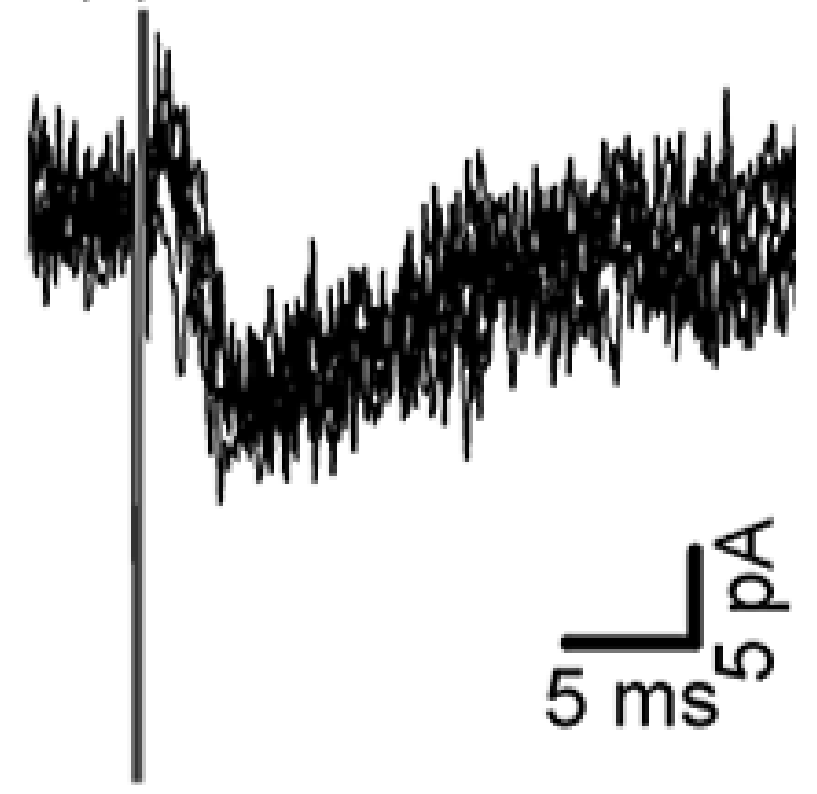

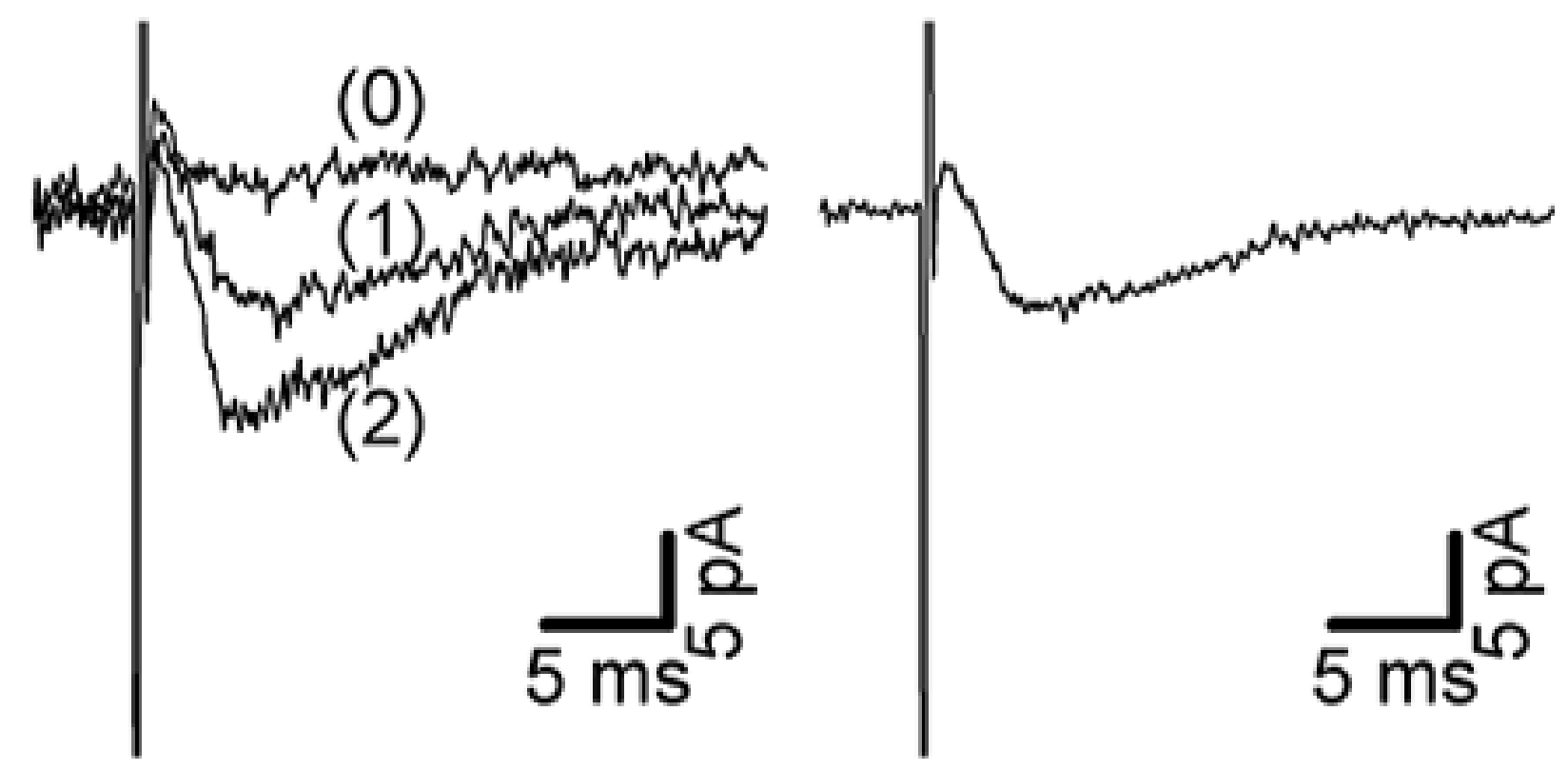



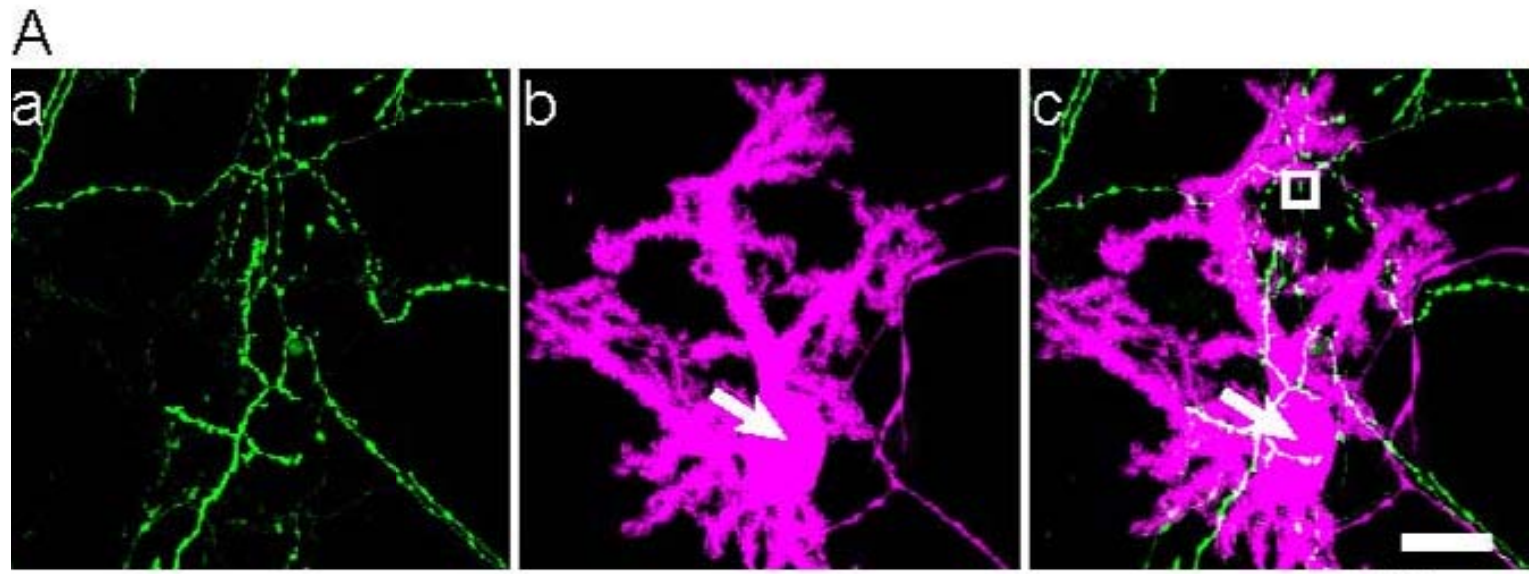

B
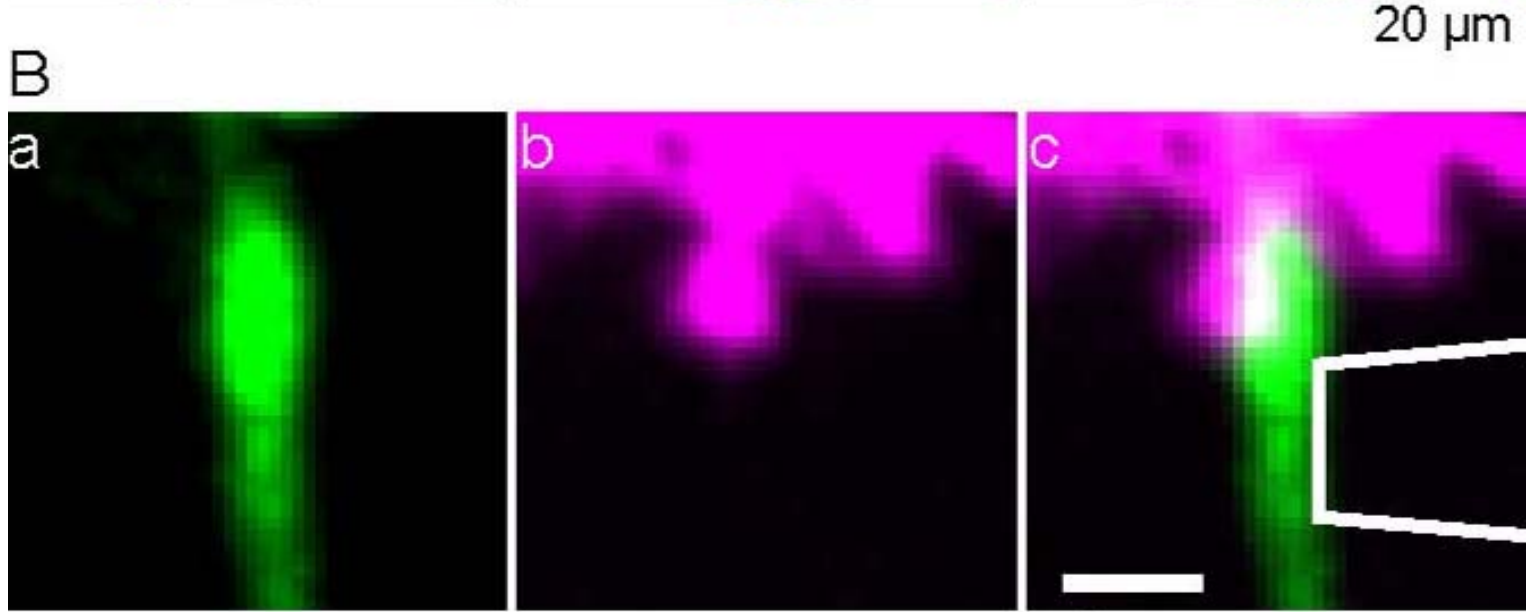

C

D

E
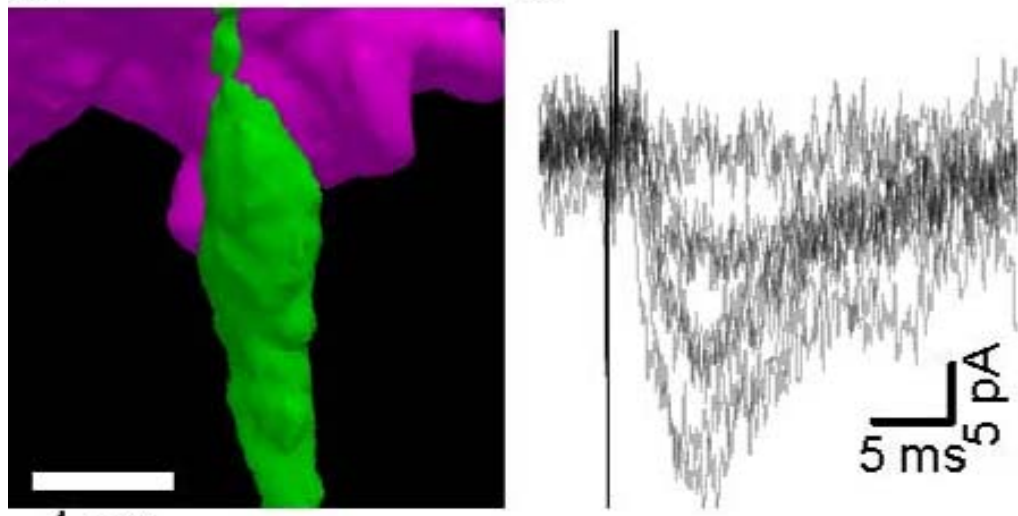

$1 \mu \mathrm{m}$

$1 \mu \mathrm{m}$

F

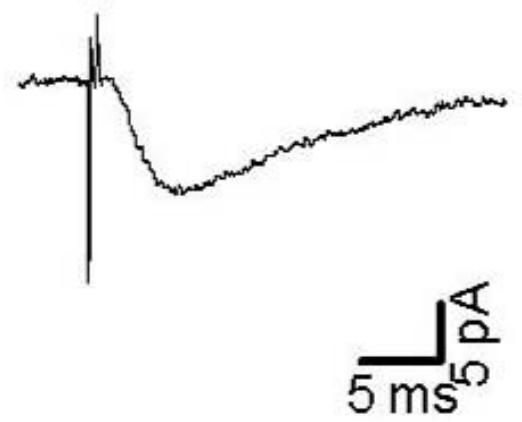

Figure 4 

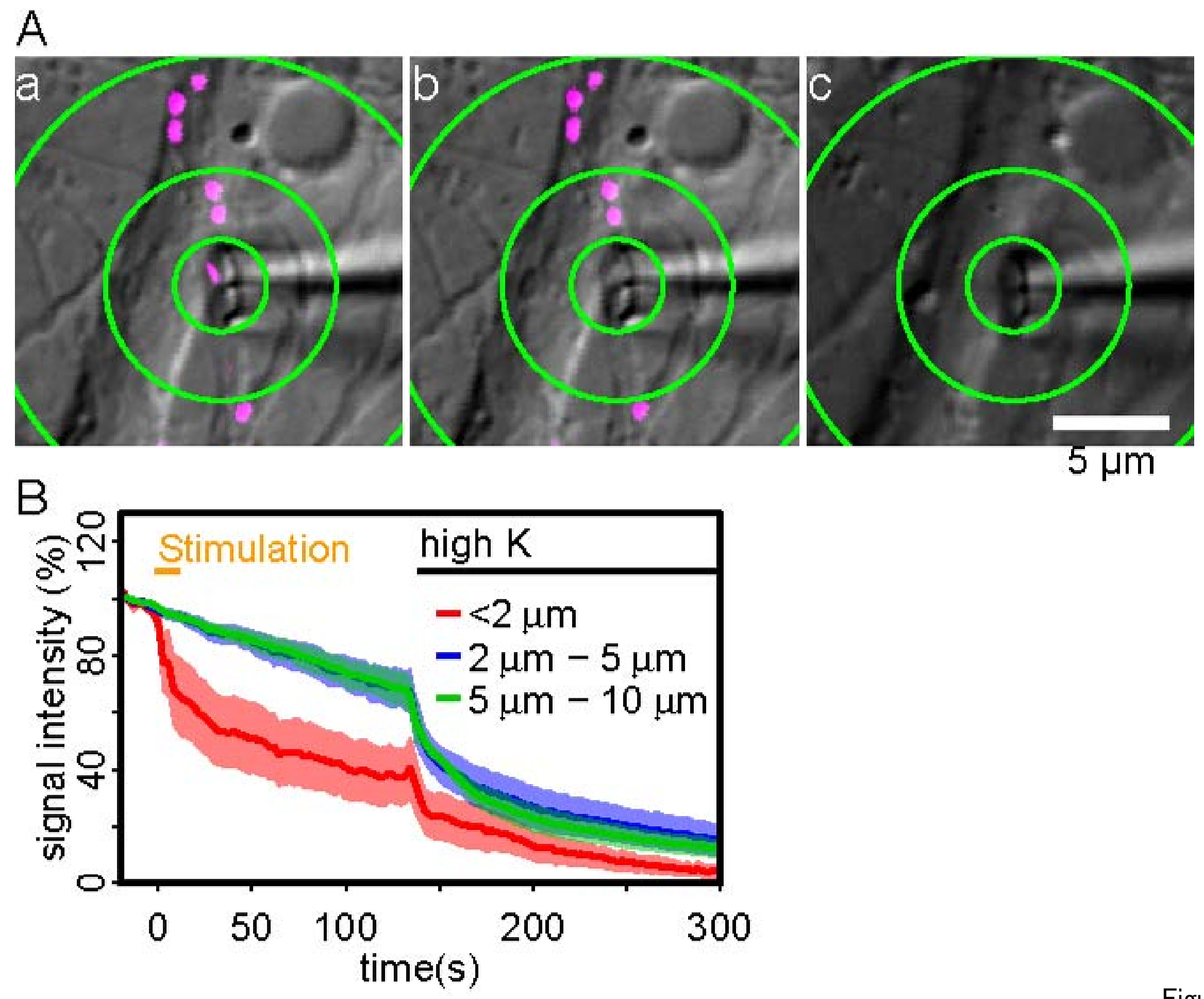

Figure 5 

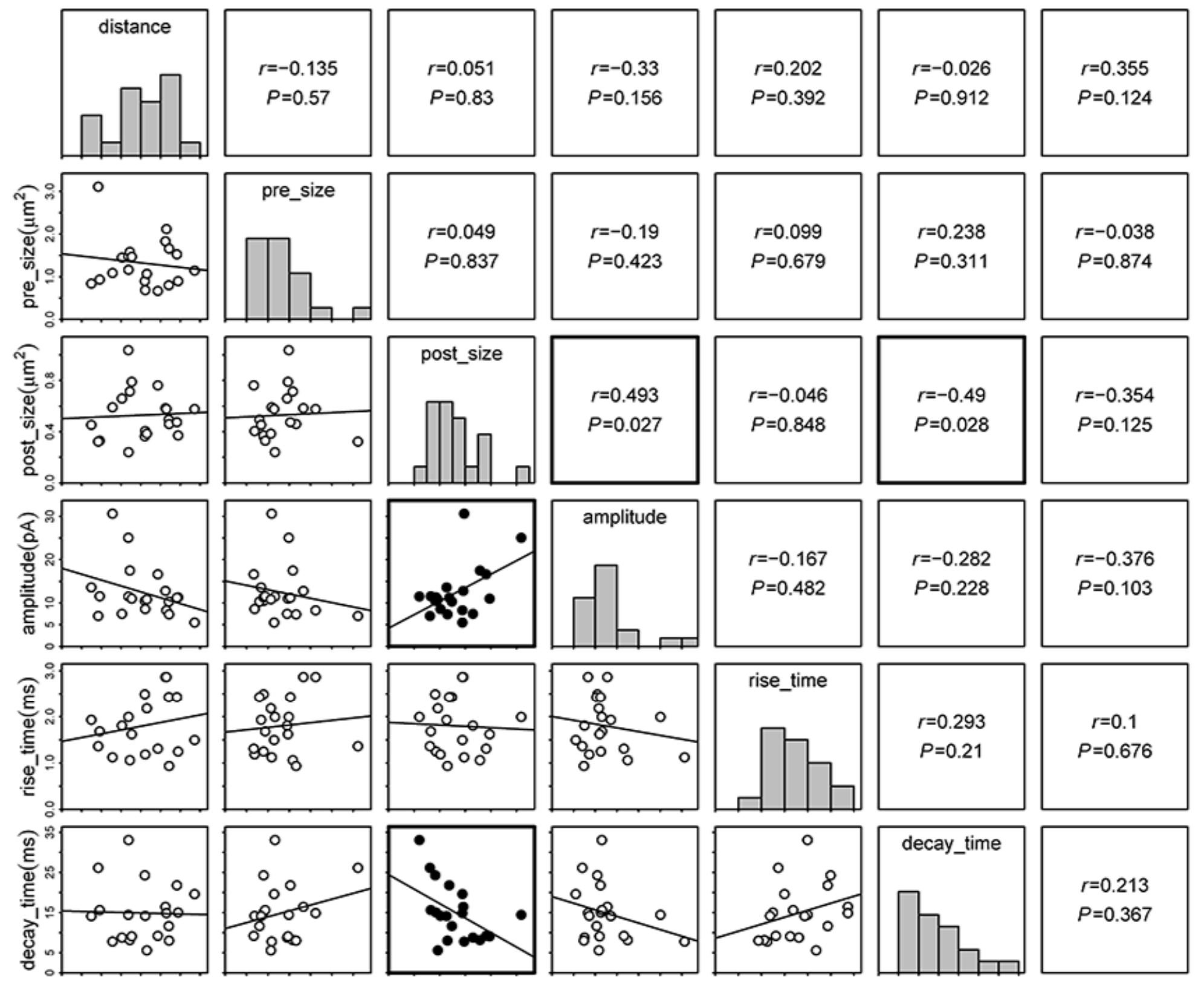

$r=0.213$

$P=0.367$
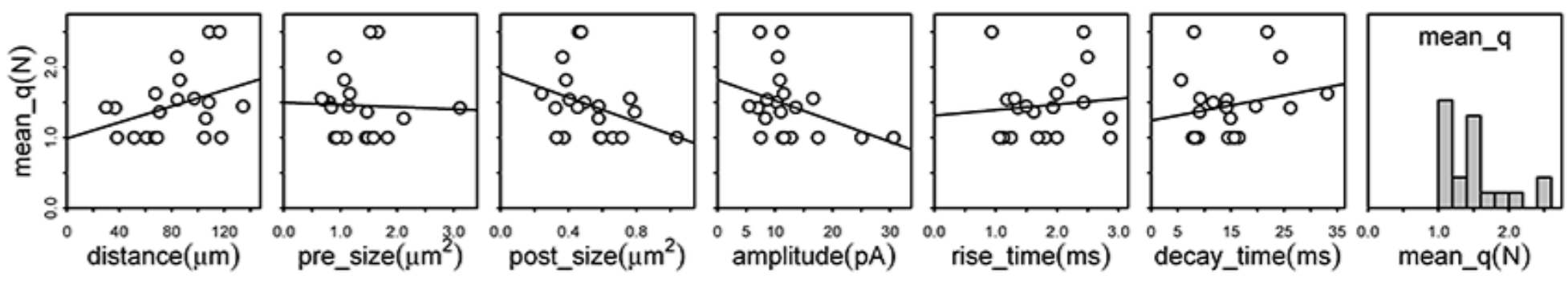

Figure 6 

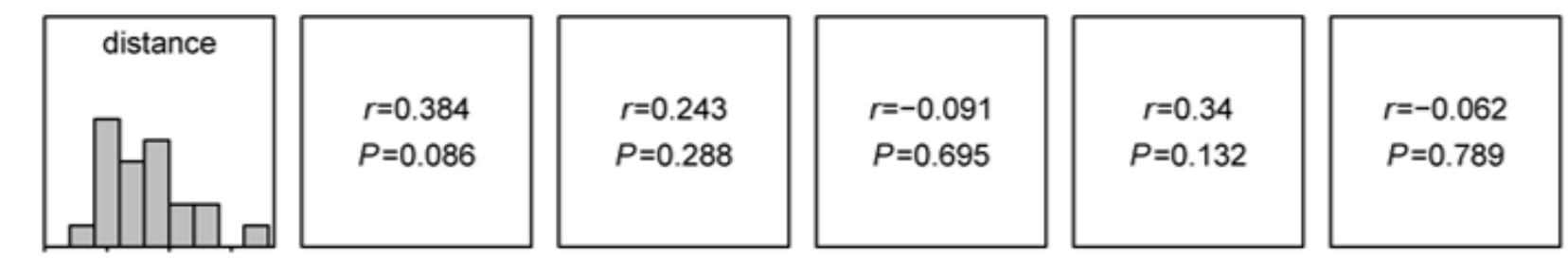

$r=0.186$

$P=0.42$
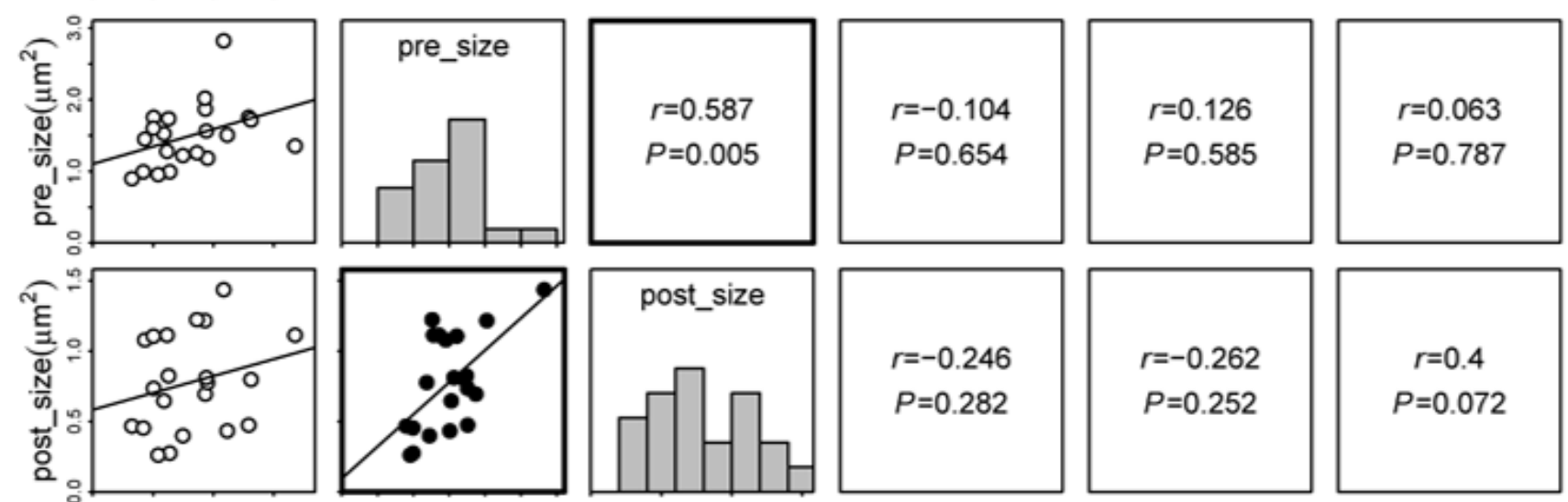

$r=0.444$

$P=0.044$
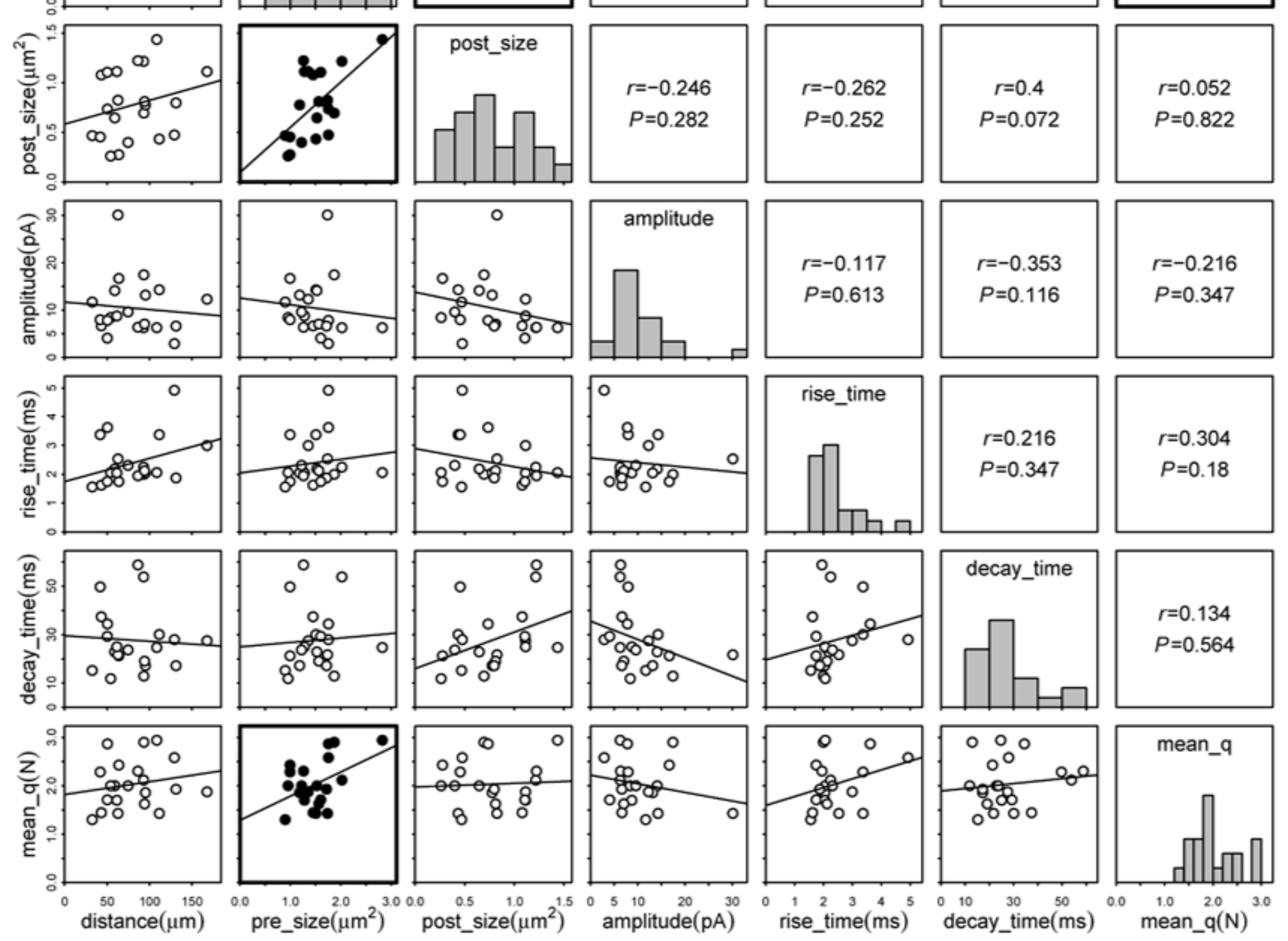

Figure 7 